\title{
Semigroup approach for identification of the unknown diffusion coefficient in a linear parabolic equation with mixed output data
}

Ebru Ozbilge ${ }^{* *}$ and Ali Demir ${ }^{2}$

\section{"Correspondence:}

ebru.ozbilge@ieu.edu.tr

'Department of Mathematics,

Faculty of Science and Literature,

Izmir University of Economics,

Sakarya Caddesi, No. 156, Balcova,

Izmir, 35330, Turkey

Full list of author information is

available at the end of the article

\begin{abstract}
This article presents a semigroup approach for the mathematical analysis of the inverse coefficient problems of identifying the unknown coefficient $k(x)$ in the linear parabolic equation $u_{t}(x, t)=\left(k(x) u_{x}(x, t)\right)_{x}$ with mixed boundary conditions $k(0) u_{x}(0, t)=\psi_{0}, u(1, t)=\psi_{1}$. The aim of this paper is to investigate the distinguishability of the input-output mappings $\Phi[\cdot]: \mathcal{K} \rightarrow H^{1,2}[0, T]$,

$\Psi[\cdot]: \mathcal{K} \rightarrow H^{1,2}[0, T]$ via semigroup theory. In this paper, we show that if the null space of the semigroup $T(t)$ consists of only zero function, then the input-output mappings $\Phi[\cdot]$ and $\Psi[\cdot]$ have the distinguishability property. It is shown that the types of the boundary conditions and the region on which the problem is defined have a significant impact on the distinguishability property of these mappings. Moreover, in the light of measured output data (boundary observations) $f(t):=u(0, t)$ or/and $h(t):=k(1) u_{x}(1, t)$, the values $k(0)$ and $k(1)$ of the unknown diffusion coefficient $k(x)$ at $x=0$ and $x=1$, respectively, can be determined explicitly. In addition to these, the values $k^{\prime}(0)$ and $k^{\prime}(1)$ of the unknown coefficient $k(x)$ at $x=0$ and $x=1$, respectively, are also determined via the input data. Furthermore, it is shown that measured output data $f(t)$ and $h(t)$ can be determined analytically by an integral representation. Hence the input-output mappings $\Phi[\cdot]: \mathcal{K} \rightarrow H^{1,2}[0, T]$, $\Psi[\cdot]: \mathcal{K} \rightarrow H^{1,2}[0, T]$ are given explicitly in terms of the semigroup.
\end{abstract}

\section{Introduction}

Consider the following initial boundary value problem:

$$
\left\{\begin{array}{l}
u_{t}(x, t)=\left(k(x) u_{x}(x, t)\right)_{x}, \quad(x, t) \in \Omega_{T}, \\
u(x, 0)=g(x), \quad 0<x<1, \\
k(0) u_{x}(0, t)=\psi_{0}, \quad u(1, t)=\psi_{1}, \quad 0<t<T,
\end{array}\right.
$$

where $\Omega_{T}=\left\{(x, t) \in R^{2}: 0<x<1,0<t \leq T\right\}$. The left flux $\psi_{0}$ and the right boundary condition $\psi_{1}$ are assumed to be constants. The functions $c_{1}>k(x) \geq c_{0}>0$ and $g(x)$ satisfy the following conditions:

(C1) $k(x) \in H^{1,2}[0,1]$;

(C2) $g(x) \in H^{2,2}[0,1], g^{\prime}(0)=\psi_{0}, g(1)=\psi_{1}$.

Under these conditions, the initial boundary value problem (1) has the unique solution $u(x, t) \in H^{2,2}[0,1] \cap H^{1,2}[0,1][1-4]$.

\section{Springer}

O 2013 Ozbilge and Demir; licensee Springer. This is an Open Access article distributed under the terms of the Creative Commons Attribution License (http://creativecommons.org/licenses/by/2.0), which permits unrestricted use, distribution, and reproduction in any medium, provided the original work is properly cited. 
Consider the inverse problem of determining the unknown coefficient $k=k(x)$ [5-9] from the following observations at the boundaries $x=0$ and $x=1$ :

$$
u(0, t)=f(t), \quad k(1) u_{x}(1, t)=h(t), \quad t \in(0, T] .
$$

Here $u=u(x, t)$ is the solution of the parabolic problem (1). The functions $f(t), h(t)$ are assumed to be noisy free measured output data. In this context, the parabolic problem (1) will be referred to as a direct (forward) problem with the inputs $g(x)$ and $k(x)$. It is assumed that the functions $f(t)$ and $h(t)$ belong to $H^{1,2}[0, T]$ and satisfy the consistency conditions $f(0)=g(0), h^{\prime}(0)=k(1) g^{\prime}(1)$.

We denote by $\mathcal{K}:=\left\{k(x) \in H^{1,2}[0,1]: c_{1}>k(x) \geq c_{0}>0, x \in[0,1]\right\} \subset H^{1,2}[0,1]$, the set of admissible coefficients $k=k(x)$ and introduce the input-output mappings $\Phi[\cdot]: \mathcal{K} \rightarrow$ $H^{1,2}[0, T], \Psi[\cdot]: \mathcal{K} \rightarrow H^{1,2}[0, T]$, where

$$
\Phi[k]=\left.u(x, t ; k)\right|_{x=0}, \quad \Psi[k]=\left.k(x) u_{x}(x, t ; k)\right|_{x=1}, \quad k \in \mathcal{K}, f(t), h(t) \in H^{1,2}[0, T] .
$$

Then the inverse problem [10] with the measured data $f(t)$ and $h(t)$ can be formulated as the following operator equations:

$$
\Phi[k]=f, \quad \Psi[k]=h, \quad k \in \mathcal{K}, f, h \in H^{1,2}[0, T] .
$$

We denote by $\mathcal{K}:=\left\{k(x) \in H^{1,2}[0,1]: c_{1}>k(x) \geq c_{0}>0, x \in[0,1]\right\} \subset H^{1,2}[0,1]$, the set of admissible coefficients $k=k(x)$. The monotonicity, continuity and hence invertibility of the input-output mappings $\Phi[\cdot]: \mathcal{K} \rightarrow H^{1,2}[0, T]$ and $\Psi[\cdot]: \mathcal{K} \rightarrow H^{1,2}[0, T]$ are given in $[3,4]$.

The aim of this paper is to study a distinguishability of the unknown coefficient via the above input-output mappings. We say that the mapping $\Phi[\cdot]: \mathcal{K} \rightarrow H^{1,2}[0, T]$ (or $\left.\Psi[\cdot]: \mathcal{K} \rightarrow H^{1,2}[0, T]\right)$ has the distinguishability property if $\Phi\left[k_{1}\right] \neq \Phi\left[k_{2}\right]\left(\Psi\left[k_{1}\right] \neq \Psi\left[k_{2}\right]\right)$ implies $k_{1}(x) \neq k_{2}(x)$. This, in particular, means injectivity of the inverse mappings $\Phi^{-1}$ and $\Psi^{-1}$.

The purpose of this paper is to study the distinguishability of the unknown coefficient via the above input-output mappings. The results presented here are the first ones, to the knowledge of authors, from the point of view of semigroup approach [11] to inverse problems. This approach sheds more light on the identifiability of the unknown coefficient [12] and shows how much information can be extracted from the measured output data, in particular in the case of constant flux and boundary data [12-15].

The paper is organized as follows. In Section 2, the analysis of the semigroup approach is given for the inverse problem with the measured data $f(t)$. A similar analysis is applied to the inverse problem with the single measured output data $h(t)$ given at the point $x=$ 1 in Section 3. The inverse problem with two Neumann measured data $f(t)$ and $h(t)$ is discussed in Section 4. Finally, some concluding remarks are given in Section 5.

\section{Analysis of the inverse problem with measured output data $f(t)$}

Consider now the inverse problem with one measured output data $f(t)$ at $x=0$. In order to formulate the solution of the parabolic problem (1) in terms of a semigroup, let us first 
arrange the parabolic equation as follows:

$$
u_{t}(x, t)-\left(k(0) u_{x}(x, t)\right)_{x}=\left((k(x)-k(0)) u_{x}(x, t)\right)_{x}, \quad(x, t) \in \Omega_{T} .
$$

Then the initial boundary value problem (1) can be rewritten in the following form:

$$
\begin{aligned}
& u_{t}(x, t)-\left(k(0) u_{x}(x, t)\right)_{x}=\left((k(x)-k(0)) u_{x}(x, t)\right)_{x}, \quad(x, t) \in \Omega_{T}, \\
& u(x, 0)=g(x), \quad 0<x<1, \\
& k(0) u_{x}(0, t)=\psi_{0}, \quad u(1, t)=\psi_{1}, \quad 0<t<T .
\end{aligned}
$$

Here we assume that $k(0)$ was known. Later we will determine the value $k(0)$. In order to formulate the solution of the parabolic problem (5) in terms of a semigroup, we need to define the following function:

$$
v(x, t)=u(x, t)-\frac{\psi_{0}}{k(0)} x+\psi_{0}-\psi_{1}, \quad x \in[0,1]
$$

which satisfies the following parabolic problem:

$$
\begin{aligned}
& v_{t}(x, t)+A[v(x, t)]=\left((k(x)-k(0))\left(v_{x}(x, t)+\frac{\psi_{0}}{k(0)}\right)\right)_{x}, \quad(x, t) \in \Omega_{T}, \\
& v(x, 0)=g(x)-\frac{\psi_{0}}{k(0)} x+\psi_{0}-\psi_{1}, \quad 0<x<1, \\
& k(0) v_{x}(0, t)=0, \quad v(1, t)=0, \quad 0<t<T .
\end{aligned}
$$

Here $A[v(x, t)]:=-k(0) d^{2} v(x, t) / d x^{2}$ is a second-order differential operator, its domain is $D_{A}=\left\{u \in H^{2,2}(0,1) \cap H^{1,2}[0,1]: u_{x}(0)=u(1)=0\right\}$. Since the initial value function $g(x)$ belongs to $C^{2}[0,1]$, it is obvious that $g(x) \in D_{A}$.

Denote by $T(t)$ the semigroup of linear operators generated by the operator $-A[5,6]$. Note that we can easily find the eigenvalues and eigenfunctions of the differential operator $A$. Furthermore, the semigroup $T(t)$ can be easily constructed by using the eigenvalues and eigenfunctions of a differential operator $A$. For this reason, we first consider the following eigenvalue problem:

$$
\begin{aligned}
& A \phi(x)=\lambda \phi(x), \\
& \phi_{x}(0)=0 ; \quad \phi(1)=0 .
\end{aligned}
$$

This problem is called a Sturm-Liouville problem. We can easily determine that the eigenvalues are $\lambda_{n}=k(0)(2 n-1)^{2} \pi^{2} / 4$ for all $n=1, \ldots$ and the corresponding eigenfunctions are $\phi_{n}(x)=\sqrt{2} \cos ((2 n-1) x \pi / 2)$. In this case, the semigroup $T(t)$ can be represented in the following way:

$$
T(t) U(x, s)=\sum_{n=0}^{\infty}\left\langle\phi_{n}(x), U(x, s)\right| e^{-\lambda_{n} t} \phi_{n}(x),
$$


where $\left\langle\phi_{n}(x), U(x, s)\right\rangle=\int_{0}^{1} \phi_{n}(x) U(x, s) d x$. The null space of the semigroup $T(t)$ of the linear operators can be defined as follows:

$$
N(T)=\left\{U(x, s):\left\langle\phi_{n}(x), U(x, s)\right\rangle=0 \text {, for all } n=1,2,3, \ldots\right\} .
$$

From the definition of the semigroup $T(t)$, we can say that the null space of it is an empty set, i.e., $N(T)=\{0\}$. This result is very important for the uniqueness of the unknown coefficient $k(x)$.

The unique solution of the initial value problem (7) in terms of a semigroup $T(t)$ can be represented in the following form:

$$
v(x, t)=T(t) v(x, 0)+\int_{0}^{t} T(t-s)\left((k(x)-k(0))\left(v_{x}(x, t)+\frac{\psi_{0}}{k(0)}\right)\right)_{x} d s .
$$

Hence, by using identity (6), the solution $u(x, t)$ of the parabolic problem (5) in terms of a semigroup can be written in the following form:

$$
\begin{aligned}
u(x, t)= & \frac{\psi_{0}}{k(0)} x+\psi_{1}-\psi_{0}+T(t)\left(g(x)-\frac{\psi_{0}}{k(0)} x+\psi_{0}-\psi_{1}\right) \\
& +\int_{0}^{t} T(t-s)\left((k(x)-k(0)) u_{x}(x, s)\right)_{x} d s .
\end{aligned}
$$

In order to arrange the above solution representation, let us define the following:

$$
\begin{aligned}
& \zeta(x)=\left(g(x)-\frac{\psi_{0}}{k(0)} x+\psi_{0}-\psi_{1}\right), \\
& \xi(x, t)=\left((k(x)-k(0)) u_{x}(x, s)\right)_{x}, \\
& z(x, t)=\sum_{n=0}^{\infty}\left\langle\phi_{n}(x), \zeta(x)\right\rangle e^{-\lambda_{n} t} \phi_{n}^{\prime}(x), \\
& w(x, t, s)=\sum_{n=0}^{\infty}\left\langle\phi_{n}(x), \xi(x, s)\right\rangle e^{-\lambda_{n} t} \phi_{n}^{\prime}(x) .
\end{aligned}
$$

Then we can rewrite the solution representation in terms of $\zeta(x)$ and $\xi(x, s)$ in the following form:

$$
u(x, t)=\frac{\psi_{0}}{k(0)} x+\psi_{1}-\psi_{0}+T(t) \zeta(x)+\int_{0}^{t} T(t-s) \xi(x, s) d s .
$$

Substituting $x=0$ into this solution representation yields

$$
u(0, t)=\psi_{1}-\psi_{0}+T(t) \zeta(0)+\int_{0}^{t} T(t-s) \xi(0, s) d s
$$

Taking into account the overmeasured data $u(0, t)=f(t)$, we get

$$
f(t)=\left(\psi_{1}-\psi_{0}+T(t) \zeta(0)+\int_{0}^{t} T(t-s) \xi(0, s) d s\right),
$$

which implies that $f(t)$ can be determined analytically. 
Differentiating both sides of the above identity with respect to $x$ and using semigroup properties at $x=0$ yield

$$
u_{x}(0, t)=\frac{\psi_{0}}{k(0)}+z(0, t)+\int_{0}^{t} w(0, t-s, s) d s
$$

Using the boundary condition $k(0) u_{x}(0, t)=\psi_{0}$, we can write $k(0)=\psi_{0} / u_{x}(0, t)$ for all $t \geq 0$ which can be rewritten in terms of a semigroup in the following form:

$$
k(0)=\psi_{0} /\left(-\psi_{0}+\psi_{1}+z(0, t)+\int_{0}^{t} w(0, t-s, s) d s\right) .
$$

Taking limit as $t \rightarrow 0$ in the above identity, we obtain the following explicit formula for the value $k(0)$ of the unknown coefficient $k(x)$ :

$$
k(0)=\psi_{0} /\left(-\psi_{0}+\psi_{1}+z(0,0)\right) .
$$

The right-hand side of identity (11) defines explicitly the semigroup representation of the input-output mapping $\Phi[k]$ on the set of admissible unknown diffusion coefficients $\mathcal{K}$ :

$$
\Phi[k](x):=\psi_{1}-\psi_{0}+T(t) \zeta(0)+\int_{0}^{t} T(t-s) \xi(0, s) d s, \quad \forall t \in[0, T]
$$

Let us differentiate now both sides of identity (8) with respect to $t$ :

$$
\begin{aligned}
u_{t}(x, t)= & T(t) A\left(g(x)-\frac{\psi_{0}}{k(0)} x+\psi_{0}-\psi_{1}\right)+\left((k(x)-k(0)) u_{x}(x, t)\right)_{x} \\
& +\int_{0}^{t} A T(t-s)\left((k(x)-k(0)) u_{x}(x, s)\right)_{x} d s .
\end{aligned}
$$

Using the semigroup property $-\int_{0}^{t} A T(s) u(x, s) d s=T(t) u(x, t)-T(0) u(x, t)$, we obtain

$$
\begin{aligned}
u_{t}(x, t)= & -k(0) T(t) g^{\prime \prime}(x)-2 T(0)\left((k(x)-k(0)) u_{x}(x, t)\right)_{x} \\
& +T(t)\left((k(x)-k(0)) u_{x}(x, 0)\right)_{x} .
\end{aligned}
$$

Taking $x=0$ in the above identity, we get

$$
\begin{aligned}
u_{t}(0, t)= & -k(0) T(t) g^{\prime \prime}(0)-T(0) k^{\prime}(0) u_{x}(0,0) \\
& +T(t)\left(k^{\prime}(0) u_{x}(0,0)\right)-T(0)\left(k^{\prime}(0) u_{x}(0, t)\right) .
\end{aligned}
$$

Since $u(0, t)=f(t)$, we have $u_{t}(0, t)=f^{\prime}(t)$. Taking into account this and substituting $t=0$ yield

$$
f^{\prime}(0)=-k(0) g^{\prime \prime}(0)-k^{\prime}(0) \frac{g^{\prime}(0)}{k(0)}
$$


Solving this equation for $k^{\prime}(0)$ and substituting $u_{x}(0,0)=g^{\prime}(0) / k(0)$, we obtain the following explicit formula for the value $k^{\prime}(0)$ of the first derivative $k^{\prime}(x)$ of the unknown coefficient at $x=0$ :

$$
k^{\prime}(0)=\frac{-k^{2}(0) g^{\prime \prime}(0)-k(0) f^{\prime}(0)}{g^{\prime}(0)} .
$$

Under the determined values $k(0)$ and $k^{\prime}(0)$, the set of admissible coefficients can be defined as follows:

$$
\mathcal{K}_{0}:=\left\{k \in \mathcal{K}: k(0)=\frac{\psi_{0}}{-\psi_{0}+\psi_{1}+z(0,0)}, k^{\prime}(0)=\frac{-k^{2}(0) g^{\prime \prime}(0)-k(0) f^{\prime}(0)}{g^{\prime}(0)}\right\} .
$$

The following lemma implies the relationship between the diffusion coefficients $k_{1}(x)$, $k_{2}(x) \in \mathcal{K}$ at $x=0$ and the corresponding outputs $f_{j}(t):=u\left(0, t ; k_{j}\right), j=1,2$.

Lemma 2.1 Let $u_{1}(x, t)=u\left(x, t ; k_{1}\right)$ and $u_{2}(x, t)=u\left(x, t ; k_{2}\right)$ be solutions of the direct problem (5) corresponding to the admissible coefficients $k_{1}(x), k_{2}(x) \in \mathcal{K}$. Suppose that $f_{j}(t)=$ $u\left(0, t ; k_{j}\right), j=1,2$, are the corresponding outputs and denote by $\Delta f(t)=f_{1}(t)-f_{2}(t), \Delta \xi(x, t)=$ $\xi^{1}(x, t)-\xi^{2}(x, t)$. If the condition

$$
k_{1}(0)=k_{2}(0):=k(0)
$$

holds, then the outputs $f_{j}(t), j=1,2$, satisfy the following integral identity:

$$
\Delta f(\tau)=\int_{0}^{\tau} T(\tau-s) \Delta \xi(0, s) d s d s
$$

for each $\tau \in(0, T]$.

Proof The solutions of the direct problem (5) corresponding to the admissible coefficients $k_{1}(x), k_{2}(x) \in \mathcal{K}$ can be written at $x=0$ as follows:

$$
\begin{aligned}
& f_{1}(\tau)=\psi_{1}-\psi_{0}+T(t) \zeta^{1}(0)+\int_{0}^{t} T(t-s) \xi^{1}(0, s) d s \\
& f_{2}(\tau)=\psi_{1}-\psi_{0}+T(t) \zeta^{2}(0)+\int_{0}^{t} T(t-s) \xi^{2}(0, s) d s
\end{aligned}
$$

respectively, by using representation (11). From identity (9) it is obvious that $\zeta^{1}(0, \tau)=$ $\zeta^{2}(0, \tau)$ for each $\tau \in(0, T]$. Hence the difference of these formulas implies the desired result.

This lemma with identity (14) implies the following.

Corollary 2.1 Let conditions of Lemma 2.1 hold. Then $f_{1}(t)=f_{2}(t), \forall t \in[0, T]$, if and only if

$$
\left\langle\phi_{n}(x), \Delta \xi(0, s)\right\rangle=\left\langle\phi_{n}(x), \xi^{1}(x, t)-\xi^{2}(x, t)\right\rangle=0, \quad \forall t \in(0, T], n=0,1, \ldots
$$


Since the Strum-Liouville problem generates a complete orthogonal family of eigenfunctions, the null space of a semigroup contains only zero function, i.e., $N(T)=\{0\}$. Thus Corollary 2.1 states that $f_{1} \equiv f_{2}$ if and only if $\xi^{1}(x, t)-\xi^{2}(x, t)=0$ for all $(x, t) \in \Omega_{T}$. The definition of $\xi(x, t)$ implies that $k_{1}(x)=k_{2}(x)$ for all $x \in[0,1]$.

The combination of the conclusions of Lemma 2.1 and Corollary 2.1 can be given by the following theorem which states the distinguishability of the input-output mapping $\Phi[\cdot]: \mathcal{K}_{0} \rightarrow H^{1,2}[0, T]$.

Theorem 2.1 Let conditions (C1) and (C2) hold. Assume that $\Phi[\cdot]: \mathcal{K}_{0} \rightarrow H^{1,2}[0, T]$ is the input-output mapping defined by (3) and corresponding to the measured output $f(t):=$ $u(0, t)$. Then the mapping $\Phi[k]$ has the distinguishability property in the class of admissible coefficients $\mathcal{K}_{0}$, i.e.,

$$
\Phi\left[k_{1}\right] \neq \Phi\left[k_{2}\right] \quad \forall k_{1}, k_{2} \in \mathcal{K}_{0}, \quad k_{1}(x) \neq k_{2}(x) .
$$

\section{Analysis of the inverse problem with measured output data $h(t)$}

Consider now the inverse problem with one measured output data $h(t)$ at $x=1$. As in the previous section, let us arrange the parabolic equation as follows:

$$
u_{t}(x, t)-\left(k(1) u_{x}(x, t)\right)_{x}=\left((k(x)-k(1)) u_{x}(x, t)\right)_{x}, \quad(x, t) \in \Omega_{T} .
$$

Then the initial boundary value problem (1) can be rewritten in the following form:

$$
\begin{aligned}
& u_{t}(x, t)-\left(k(1) u_{x}(x, t)\right)_{x}=\left((k(x)-k(1)) u_{x}(x, t)\right)_{x}, \quad(x, t) \in \Omega_{T}, \\
& u(x, 0)=g(x), \quad 0<x<1, \\
& k(0) u_{x}(0, t)=\psi_{0}, \quad u(1, t)=\psi_{1}, \quad 0<t<T .
\end{aligned}
$$

In order to formulate the solution of the above parabolic problem in terms of a semigroup, let us use the same variable $v(x, t)$ in identity (6), which satisfies the following parabolic problem:

$$
\begin{aligned}
& v_{t}(x, t)+B[v(x, t)]=\left((k(x)-k(1)) u_{x}(x, t)\right)_{x}, \quad(x, t) \in \Omega_{T}, \\
& v(x, 0)=g(x)-\frac{\psi_{0}}{k(0)} x+\frac{\psi_{0}}{k(0)}-\psi_{1}, \quad 0<x<1, \\
& k(0) v_{x}(0, t)=0, \quad v(1, t)=0, \quad 0<t<T .
\end{aligned}
$$

Here $B[v(x, t)]:=-k(1) d^{2} v(x, t) / d x^{2}$ is a second-order differential operator, its domain is $D_{B}=\left\{u \in H^{2,2}(0,1) \cap H^{1,2}[0,1]: u_{x}(0)=u(1)=0\right\}$. Since the initial value function $g(x)$ belongs to $H^{2,2}[0,1]$, it is obvious that $g(x) \in D_{B}$.

Denote by $S(t)$ the semigroup of linear operators generated by the operator $-A[5,6]$. As in the previous section, we can easily find the eigenvalues and eigenfunctions of the differential operator $B$. Furthermore, the semigroup $S(t)$ can be easily constructed by using the eigenvalues and eigenfunctions of the differential operator $B$. For this reason, we first consider the following eigenvalue problem:

$$
B \phi(x)=\lambda \phi(x), \quad \phi_{x}(0)=0 ; \quad \phi(1)=0 .
$$


This problem is called a Sturm-Liouville problem. We can easily determine that the eigenvalues are $\lambda_{n}=k(1)(2 n-1)^{2} \pi^{2} / 4$ for all $n=0,1, \ldots$ and the corresponding eigenfunctions become $\phi_{n}(x)=\sqrt{2} \cos ((2 n-1) \pi / 2 x)$. Hence the semigroup $S(t)$ can be represented in the following form:

$$
S(t) U(x, s)=\sum_{n=0}^{\infty}\left\langle\phi_{n}(x), U(x, s)\right\rangle e^{-\lambda_{n} t} \phi_{n}(x)
$$

The null space of the semigroup $S(t)$ of the linear operators can be defined as follows:

$$
N(S)=\left\{U(x, s):\left\langle\phi_{n}(x), U(x, s)\right\rangle=0, \text { for all } n=1,2,3, \ldots\right\}
$$

Since the Sturm-Liouville problem generates a complete orthogonal family of eigenfunctions, we can say that the null space of the semigroup $S(t)$ is an empty set, i.e., $N(S)=\emptyset$. This result is very important for the uniqueness of the unknown coefficient $k(x)$.

The unique solution of the initial value problem (16) in terms of a semigroup $S(t)$ can be represented in the following form:

$$
v(x, t)=S(t) v(x, 0)+\int_{0}^{t} S(t-s)\left((k(x)-k(1))\left(v_{x}(x, s)+\frac{\psi_{0}}{k(0)}\right)\right)_{x} d s .
$$

Hence, by using identity (6), the solution $u(x, t)$ of the parabolic problem (15) in terms of a semigroup can be written in the following form:

$$
\begin{aligned}
u(x, t)= & \frac{\psi_{0}}{k(0)} x+\psi_{1}-\frac{\psi_{0}}{k(0)}+S(t)\left(g(x)-\frac{\psi_{0}}{k(0)} x+\frac{\psi_{0}}{k(0)}-\psi_{1}\right) \\
& +\int_{0}^{t} S(t-s)\left((k(x)-k(1)) u_{x}(x, s)\right)_{x} d s .
\end{aligned}
$$

Defining the following:

$$
\begin{aligned}
& \zeta(x)=\left(g(x)-\frac{\psi_{0}}{k(0)} x+\frac{\psi_{0}}{k(0)}-\psi_{1}\right), \\
& \chi(x, s)=\left((k(x)-k(1)) u_{x}(x, s)\right)_{x}, \\
& z_{1}(x, t)=\sum_{n=0}^{\infty}\left\langle\phi_{n}(x), \zeta(x)\right\rangle e^{-\lambda_{n} t} \phi_{n}^{\prime}(x), \\
& w_{1}(x, t, s)=\sum_{n=0}^{\infty}\left\langle\phi_{n}(x), \chi(x, s)\right\rangle e^{-\lambda_{n} t} \phi_{n}^{\prime}(x) .
\end{aligned}
$$

The solution representation of the parabolic problem (17) can be rewritten in the following form:

$$
u(x, t)=\frac{\psi_{0}}{k(0)} x+\psi_{1}-\frac{\psi_{0}}{k(0)}+S(t) \zeta(x)+\int_{0}^{t} S(t-s) \chi(x, s) d s
$$


Differentiating both sides of the above identity with respect to $x$ and substituting $x=1$ yield

$$
u_{x}(1, t)=\frac{\psi_{0}}{k(0)}+z_{1}(1, t)+\int_{0}^{t} w_{1}(1, t-s, s) d s
$$

Taking into account the overmeasured data $k(1) u_{x}(1, t)=h(t)$, we get

$$
h(t)=k(1)\left(\frac{\psi_{0}}{k(0)}+z_{1}(1, t)+\int_{0}^{t} w_{1}(1, t-s, s) d s\right) .
$$

Now we can determine the value $k(1)$. From the overmeasured data $k(1) u_{x}(1, t)=h(t)$, the identity $k(1)=h(t) / u_{x}(1, t)$ for all $t>0$ can be rewritten in terms of a semigroup in the following form:

$$
k(1)=\frac{h(t)}{\left(\frac{\psi_{0}}{k(0)}+z_{1}(1, t)+\int_{0}^{t} w_{1}(1, t-s, s) d s\right)} .
$$

Taking limit as $t \rightarrow 0$ in the above identity yields

$$
k(1)=h(0) /\left(\frac{\psi_{0}}{k(0)}+z_{1}(1,0)\right) .
$$

The right-hand side of the above identity defines the semigroup representation of the input-output mapping $\Psi[k]$ on the set of admissible unknown diffusion coefficient $\mathcal{K}$ :

$$
\Psi[k](x):=k(1)\left(\frac{\psi_{0}}{k(0)}+z_{1}(1, t)+\int_{0}^{t} w_{1}(1, t-s, s) d s\right), \quad \forall t \in[0, T] .
$$

Differentiating both sides of identity (17) with respect to $t$, we get

$$
\begin{aligned}
u_{t}(x, t)= & S(t) B\left(g(x)-\frac{\psi_{0}}{k(0)} x+\frac{\psi_{0}}{k(0)}-\psi_{1}\right) \\
& -S(0)\left((k(x)-k(1)) u_{x}(x, t)\right)_{x}+\int_{0}^{t} B S(t-s)\left((k(x)-k(1)) u_{x}(x, s)\right)_{x} d s .
\end{aligned}
$$

Using semigroup properties, we obtain

$$
\begin{aligned}
u_{t}(x, t)= & -S(t) k(1) g^{\prime \prime}(x)-S(0)\left(k^{\prime}(x) u_{x}(x, t)+(k(x)-k(1)) u_{x x}(x, t)\right) \\
& +S(t)\left((k(x)-k(1)) u_{x}(x, 0)\right)_{x}-S(0)\left((k(x)-k(1)) u_{x}(x, t)\right)_{x} .
\end{aligned}
$$

Taking $x=1$ in the above identity, we get

$$
u_{t}(1, t)=-S(t) k(1) g^{\prime \prime}(1)-2 S(0) k^{\prime}(1) u_{x}(1, t)+S(t) k^{\prime}(1) u_{x}(1,0) .
$$

Since $u(1, t)=\psi_{1}$, we have $u_{t}(1, t)=0$. Taking into account this and substituting $t=0$, we get

$$
0=-k(1) g^{\prime \prime}(1)-k^{\prime}(1) u_{x}(1,0) .
$$


Solving this equation for $k^{\prime}(1)$ and substituting $u_{x}(1,0)=h(0) / k(1)$, we reach the following result:

$$
k^{\prime}(1)=-\frac{k^{2}(1) g^{\prime \prime}(1)}{h(0)}
$$

Then we can define the admissible set of diffusion coefficients as follows:

$$
\mathcal{K}_{1}:=\left\{k \in \mathcal{K}: k(1)=\frac{h(0)}{\left(\frac{\psi_{0}}{k(0)}+z_{1}(1,0)\right)}, k^{\prime}(1)=-\frac{k^{2}(1) g^{\prime \prime}(1)}{h(0)}\right\} .
$$

The following lemma implies the relation between the coefficients $k_{1}(x), k_{2}(x) \in \mathcal{K}$ at $x=1$ and the corresponding outputs $h_{j}(t):=k_{j}(1) u_{x}\left(1, t ; k_{j}\right), j=1,2$.

Lemma 3.1 Let $u_{1}(x, t)=u\left(x, t ; k_{1}\right)$ and $u_{2}(x, t)=u\left(x, t ; k_{2}\right)$ be solutions of the direct problem (16) corresponding to the admissible coefficients $k_{1}(x), k_{2}(x) \in \mathcal{K}$. Suppose that $h_{j}(t)=$ $u\left(1, t ; k_{j}\right), j=1,2$, are the corresponding outputs and denote by $\Delta h(t)=h_{1}(t)-h_{2}(t)$, $\Delta w_{1}(x, t, s)=w_{1}^{1}(x, t, s)-w_{1}^{2}(x, t, s)$. If the condition

$$
k_{1}(1)=k_{2}(1):=k(1)
$$

holds, then the outputs $h_{j}(t), j=1,2$, satisfy the following integral identity:

$$
\Delta h(\tau)=k(1) \int_{0}^{\tau} \Delta w_{1}(1, \tau-s, s) d s d s
$$

for each $\tau \in(0, T]$.

Proof The solutions of the direct problem (15) corresponding to the admissible coefficients $k_{1}(x), k_{2}(x) \in \mathcal{K}$ can be written at $x=1$ as follows:

$$
\begin{aligned}
& h_{1}(\tau)=k(1)\left(\frac{\psi_{0}}{k(0)}+z_{1}^{1}(1, \tau)+\int_{0}^{t} w_{1}^{1}(1, \tau-s, s) d s\right), \\
& h_{2}(\tau)=k(1)\left(\frac{\psi_{0}}{k(0)}+z_{1}^{2}(1, \tau)+\int_{0}^{t} w_{1}^{2}(1, \tau-s, s) d s\right),
\end{aligned}
$$

respectively, by using formula (20). From definition (18), it is obvious that $z_{1}^{1}(1, \tau)=z_{1}^{2}(1, \tau)$ for each $\tau \in(0, T]$. Hence the difference of these formulas implies the desired result.

This lemma with identity (23) implies the following conclusion.

Corollary 3.1 Let the conditions of Lemma 3.1 hold. Then $h_{1}(t)=h_{2}(t), \forall t \in[0, T]$, if and only if

$$
\left\langle\phi_{n}(x), \chi^{1}(x, t)-\chi^{2}(x, t)\right\rangle=0, \quad \forall t \in(0, T], n=0,1, \ldots
$$

hold. 
Since the null space of it consists of only zero function, i.e., $N(S)=\{0\}$, Corollary 3.1 states that $h_{1} \equiv h_{2}$ if and only if $\chi^{1}(x, t)-\chi^{2}(x, t)=0$ for all $(x, t) \in \Omega_{T}$. The definition of $\chi(x, t)$ implies that $k_{1}(x)=k_{2}(x)$ for all $x \in(0,1]$.

Theorem 3.1 Let conditions (C1) and (C2) hold. Assume that $\Psi[\cdot]: \mathcal{K}_{1} \rightarrow C^{1}[0, T]$ is the input-output mapping defined by (3) and corresponding to the measured output $h(t):=$ $k(1) u_{x}(1, t)$. Then the mapping $\Psi[k]$ has the distinguishability property in the class of admissible coefficients $\mathcal{K}_{1}$, i.e.,

$$
\Psi\left[k_{1}\right] \neq \Psi\left[k_{2}\right] \quad \forall k_{1}, k_{2} \in \mathcal{K}_{1}, \quad k_{1}(x) \neq k_{2}(x) .
$$

\section{The inverse problem with mixed output data}

Consider now the inverse problem (1)-(2) with two measured output data $f(t)$ and $h(t)$. As shown before, having these two data, the values $k(0)$ as well as $k(1)$ can be defined by the above explicit formulas. Based on this result, let us define now the set of admissible coefficients $\mathcal{K}_{2}$ as an intersection:

$$
\begin{gathered}
\mathcal{K}_{2}:=\mathcal{K}_{0} \cap \mathcal{K}_{1}=\left\{k \in \mathcal{K}: k(0)=\frac{\psi_{0}}{-\psi_{0}+\psi_{1}+z(0,0)}, k(1)=\frac{h(0)}{\psi_{0} / k(0)+z_{1}(1,0)},\right. \\
\left.k^{\prime}(0)=\frac{-k^{2}(0) g^{\prime \prime}(0)-k(0) f^{\prime}(0)}{g^{\prime}(0)}, k^{\prime}(1)=\frac{-k^{2}(1) g^{\prime \prime}(1)}{h(0)}\right\} .
\end{gathered}
$$

On this set, both input-output mappings $\Phi[k]$ and $\Psi[k]$ have distinguishability property.

Corollary 4.1 The input-output mappings $\Phi[\cdot]: \mathcal{K}_{2} \rightarrow H^{1,2}[0, T]$ and $\Psi[\cdot]: \mathcal{K}_{2} \rightarrow$ $H^{1,2}[0, T]$ distinguish any two functions $k_{1}(x) \neq k_{2}(x)$ from the set $\mathcal{K}_{2}$, i.e.,

$$
\forall k_{1}(x), k_{2}(x) \in \mathcal{K}_{2}, \quad k_{1}(x) \neq k_{2}(x), \quad \Phi\left[k_{1}\right] \neq \Phi\left[k_{2}\right], \quad \Psi\left[k_{1}\right] \neq \Psi\left[k_{2}\right] .
$$

\section{Conclusion}

The aim of this study was to analyze distinguishability properties of the input-output mappings $\Phi[\cdot]: \mathcal{K}_{2} \rightarrow H^{1,2}[0, T]$ and $\Psi[\cdot]: \mathcal{K}_{2} \rightarrow H^{1,2}[0, T]$ which are naturally determined by the measured output data. In this paper we show that if the null spaces of the semigroups $T(t)$ and $S(t)$ include only zero function then the corresponding input-output mappings $\Phi[\cdot]$ and $\Psi[\cdot]$ have distinguishability property.

This study shows that boundary conditions and the region on which the problem is defined have a significant impact on the distinguishability of the input-output mappings $\Phi[\cdot]$ and $\Psi[\cdot]$ since these key elements determine the structure of the semigroups $T(t)$ and $S(t)$ of linear operators and their null spaces.

Competing interests

The authors declare that they have no competing interests.

Authors' contributions

All authors contributed equally and significantly in writing this paper. All authors read and approved the final manuscript. 


\section{Acknowledgements}

Dedicated to Professor Hari M Srivastava.

The research was supported in part by the Scientific and Technical Research Council (TUBITAK) and Izmir University of Economics.

Received: 3 January 2013 Accepted: 14 February 2013 Published: 1 March 2013

\section{References}

1. DuChateau, P: Monotonicity and invertibility of coefficient-to-data mappings for parabolic inverse problems. SIAM J. Math. Anal. 26, 1473-1487 (1995)

2. Isakov, V: On uniqueness in inverse problems for quasilinear parabolic equations. Arch. Ration. Mech. Anal. 124, 1-13 (1993)

3. Pilant, MS, Rundell, W: A uniqueness theorem for conductivity from overspecified boundary data. J. Math. Anal. Appl. 136, 20-28 (1988)

4. Renardy, M, Rogers, R: An Introduction to Partial Differential Equations. Springer, New York (2004)

5. Cannon, JR: The One-Dimensional Heat Equation. Addison-Wesley, Reading (1984)

6. DuChateau, P, Thelwell, R, Butters, G: Analysis of an adjoint problem approach to the identification of an unknown diffusion coefficient. Inverse Probl. 20,601-625 (2004)

7. Showalter, R: Monotone Operators in Banach Spaces and Nonlinear Partial Differential Equations. Am. Math. Soc., Providence (1997)

8. Hasanov, A, Demir, A, Erdem, A: Monotonicity of input-output mappings in inverse coefficient and source problem for parabolic equations. J. Math. Anal. Appl. 335, 1434-1451 (2007)

9. Hasanov, A, DuChateau, P, Pektas, B: An adjoint approach and coarse-fine mesh method for identification of the diffusion coefficient in a linear parabolic equations. J. Inverse III-Posed Probl. 14, 435-463 (2006)

10. DuChateau, P, Gottlieb, J: Introduction to Inverse Problems in Partial Differential Equations for Engineers, Physicists and Mathematicians. Kluwer Academic, Dordrecht (1996)

11. Ashyralyev, A, San, ME: An approximation of semigroup method for stochastic parabolic equations. Abstr. Appl. Anal. 2012, 684248 (2012). doi:10.1155/2012/684248

12. Demir, A, Ozbilge, E: Semigroup approach for identification of the unknown diffusion coefficient in a quasi-linear parabolic equation. Math. Methods Appl. Sci. 30, 1283-1294 (2007)

13. Ozbilge, E: Identification of the unknown diffusion coefficient in a quasi-linear parabolic equation by semigroup approach with mixed boundary conditions. Math. Methods Appl. Sci. 31, 1333-1344 (2008)

14. Demir, A, Ozbilge, E: Analysis of a semigroup approach in the inverse problem of identifying an unknown coefficient. Math. Methods Appl. Sci. 31, 1635-1645 (2008)

15. Demir, A, Hasanov, A: Identification of the unknown diffusion coefficient in a linear parabolic equation by the semigroup approach. J. Math. Anal. Appl. 340, 5-15 (2008)

\section{Submit your manuscript to a SpringerOpen ${ }^{\circ}$ journal and benefit from:}

- Convenient online submission

- Rigorous peer review

- Immediate publication on acceptance

Open access: articles freely available online

- High visibility within the field

- Retaining the copyright to your article 